\title{
Detecção Automática de Múltiplas Fraturas usando Perfilagem Geofísica com Otimização Multimodal
}

Dayara Bastos Palheta ${ }^{1}$; Carlos Eduardo Guerra ${ }^{1}$; Anderson Alvarenga de Moura Meneses ${ }^{1}$

${ }^{1}$ IEG / Instituto de Engenharia e Geociências - Universidade Federal do Oeste do Pará

Copyright 2014, SBGf - Sociedade Brasileira de Geofísica

Este texto foi preparado para a apresentação no VI Simpósio Brasileiro de Geofísica, Porto Alegre, 14 a 16 de outubro de 2014. Seu conteúdo foi revisado pelo Comitê Técnico do VI SimBGf, mas não necessariamente representa a opinião da SBGf ou de seus associados. É proibida a reprodução total ou parcial deste material para propósitos comerciais sem prévia autorização da SBGf.

\section{Resumo}

O objetivo geral da investigação deste problema é o estudo, implementação e testes de métodos de inteligência computacional, para a detecção automática de múltiplas fraturas em imagens geradas por perfilagem geofísica com base em otimização multimodal. Como resultados, apresentamos uma prova de conceito para fraturas sintéticas com o algoritmo PSO. O algoritmo é capaz de identificar as duas fraturas existentes no modelo, em múltiplas inicializações, o método de reinicialização, usado como estratégia para escapar de ótimos locais e realizar uma busca completa pelo espaço de busca é conhecido como método Multistart.

\section{Introdução}

A perfilagem geofísica, conhecida, também, como well logging foi desenvolvida por Marcel e Conrad Schlumberger em 1927 (ELLIS \& SINGER, 2007). A perfilagem determina, em geral, características estáticas das formações, tais como litologias, tipos e saturações de fluidos, porosidade, inclinação de fraturas e camadas. Um perfil de poço contém informações das rochas que se formam ao longo de um intervalo de profundidade, sendo muito relevante em geofísica para determinar as características do poço e a viabilidade econômica. Um dos avanços da perfilagem são os perfis de imagem. A imagem coletada se trata de uma imagem virtual da parede do poço. As informações mais relevantes para esse trabalho são de inclinações, tanto de camadas, quanto de ocorrências de fraturas. A descrição detalhada das fraturas pode ser obtida através de uma análise de testemunho, mas este processo é muito caro e é realizado apenas para alguns poços. Assim, a necessidade de um método computacional para resolver este problema é pertinente. Deste modo, pretende-se aplicar um método de detecção automática de múltiplas fraturas em imagens geradas por perfilagem geofísica com base em otimização multimodal, com o objetivo de encontrar todas as múltiplas soluções, já que cada uma corresponde a uma fratura.

A metodologia proposta utiliza uma abordagem baseada no método Multistart que consiste em múltiplas reinicializações, gerando soluções iniciais aleatórias, em conjunto com a otimização por enxame de partículas
(Particle Swarm Optimization, PSO), que é um método de Inteligência Computacional muito utilizado em problemas de otimização global (MENESES; MACHADO; SCHIRRU, 2009), proposto originalmente por Kennedy e Eberhart em 1995 (Kennedy e Eberhart, 1995; Eberhart e Kennedy, 2001). Neste sentido, foi desenvolvido um método baseado na otimização global para a detecção de múltiplas fraturas automatizada, visando à tomada de decisão, a fim de economizar tempo e esforço na análise de dados. O algoritmo modela uma busca colaborativa, levando em conta aspectos sociais da inteligência, além de realizar múltiplas reinicializações, como estratégia para escapar de ótimos locais e realizar uma busca completa pelo espaço de busca, esse método de reinicialização é conhecido como método Multistart. Como resultados, apresentamos uma prova de conceito para fraturas sintéticas com o algoritmo PSO. Em múltiplas inicializações, o algoritmo é capaz de identificar as duas fraturas existentes no modelo.

\section{Metodologia}

Para demonstração do método, inicialmente utilizou-se uma função de teste conhecida como função de Himmelblau, com característica multimodal e espaço de busca bidimensional (Figura 1). A função é dada pela equação 1 e os seus valores de fitnesses mínimas e os valores de mínimos estão contidos na Tabela 1. A função de Himmelblau foi implementada no Optimization Toolbox $^{\text {TM }}$ do software MATLAB ${ }^{\circledR}$.

$$
f(x, y)=\left(x^{2}+y-11\right)^{2}+\left(x+y^{2}-7\right)^{2}
$$

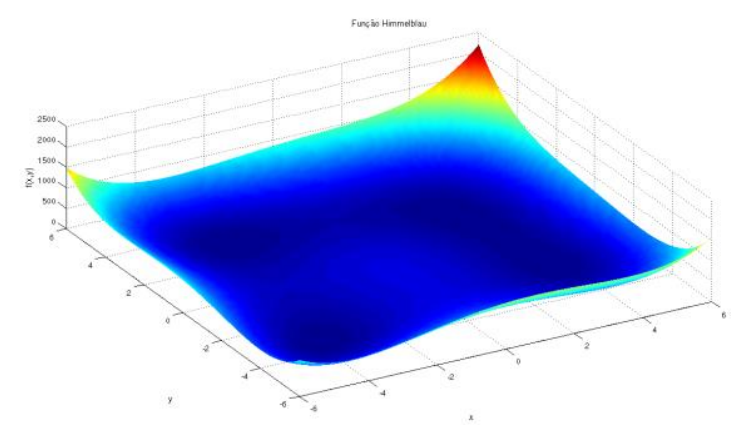

Figura 1 - Gráfico da Função de Himmelblau. 


\begin{tabular}{l|c}
\hline Coordenadas $(x, y)$ & Valores da função $f(x, y)$ \\
\hline$(3.000000,2.000000)$ & 0 \\
\hline$(-2.805118,3.131312)$ & 0 \\
\hline$(-3.779310,-3.283186)$ & $7,889 \times 10^{-31}$ \\
\hline$(3.584428,-1.848126)$ & $7,889 \times 10^{-31}$ \\
\hline
\end{tabular}

Tabela 1 - Valores das coordenadas $(x, y)$ e os valores de $\mathrm{f}(\mathrm{x}, \mathrm{y})$ dos mínimos da função de Himmelblau.

O Optimization Toolbox ${ }^{\mathrm{TM}}$ proporciona a utilização de algoritmos em grande escala de otimização. Estes algoritmos podem resolver problemas restritos e irrestritos podendo ser tanto contínuos quanto discretos. O Toolbox também inclui programação de funções de otimização multiobjetivo, que é o caso da função de Himmelblau. O objetivo era encontrar as fitnesses mínimas e os valores de mínimo da função de teste utilizando o método Multistart com a função fminunc. A função fminunc tenta encontrar o mínimo de uma função de várias variáveis, a partir de uma estimativa inicial. $\mathrm{Na}$ Tabela 2, estão contidos os melhores valores para 50 iterações. Pode-se observar que o método Multistart com a função fminunc conseguem localizar os mínimos e as fitnesses da função de Himmelblau, porém a precisão não é satisfatória.

\begin{tabular}{c|c}
\hline Valor mínimo $f(x, y)$ & Fitnesses \\
\hline$f(3.0000,2.0000)$ & $1.3219 \times \mathrm{e}^{-13}$ \\
\hline$f(3.5847,-1.8482)$ & $2.62880 \times \mathrm{e}^{-06}$ \\
\hline$f(3.6702,-1.8260)$ & 0.4148 \\
\hline$f(-2.9291,3.1493)$ & 0.5312 \\
\hline
\end{tabular}

Tabela 2 - Valores das Fitnesses mínima e os valores de mínimo da função de Himmelblau.

Em (Cardoso e Meneses, 2008) foi realizado um teste de desempenho para demonstrar o funcionamento do algoritmo PSO e apresentar seus resultados na otimização da Função de Himmelblau. Os resultados obtidos com a aplicação do PSO para a localização dos mínimos e das ftnesses da função de Himmelblau possuem maior precisão que o método aplicado no Optimization Toolbox ${ }^{T M}$ do software MATLAB ${ }^{\circledR}$, como pode ser observado na Tabela 3.

\begin{tabular}{c|c|c}
\hline $\begin{array}{c}\text { Número de } \\
\text { Iterações }\end{array}$ & Valor mínimo $f(x, y)$ & Fitnesses \\
\hline 5 & $\begin{array}{c}x=3,5785368742 \\
y=-1,8418531428\end{array}$ & 0,002134077 \\
\hline 10 & $\begin{array}{c}x=3,5818830895 \\
y=-1,8568916070\end{array}$ & 0,0016250543 \\
\hline
\end{tabular}

\begin{tabular}{l|c|c}
\hline 15 & $\begin{array}{l}x=3,5817583283 \\
y=-1,843683983\end{array}$ & 0,0005795378 \\
\hline 20 & $\begin{array}{c}x=-2,8033666030 \\
y=3,1350747506\end{array}$ & 0,0006781964 \\
\hline
\end{tabular}

Tabela 3 - Resultados obtidos com a aplicação do PSO para a Função de Himmelblau (Cardoso e Meneses, 2008).

Os resultados adquiridos com Optimization Toolbox ${ }^{\mathrm{TM}}$ do software MATLAB ${ }^{\circledR}$ demosntraram que o seu uso se torna inviável para a detecção automática de múltiplas fraturas, justificando,assim, o uso de uma metaheurística de otimização como o algoritmo PSO associado com o Método Multistart.

Para a prova de conceito, foi selecionada uma imagem (Figura 1), referida como modelo sintético (com $250 \times 337$ pixels $^{2}$ ).

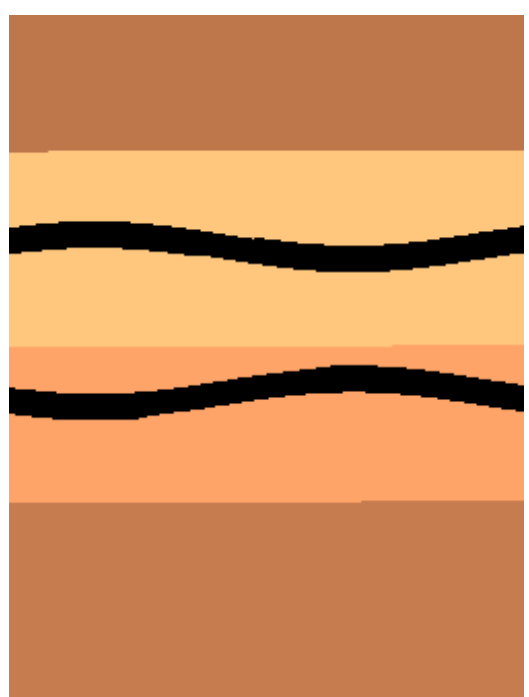

Figura 2 - O modelo sintético de levantamento geofísico 5 usado para demonstrar o método.

Um primeiro passo foi converter a imagem em tons de cinza de 8 bits. Em seguida, foram criadas imagens binarizadas com o auxílio do software MATLAB ${ }^{\circledR}$, com limiar de intensidade ajustado para 65 pixels para 0 modelo sintético 5 (Figura 3). 


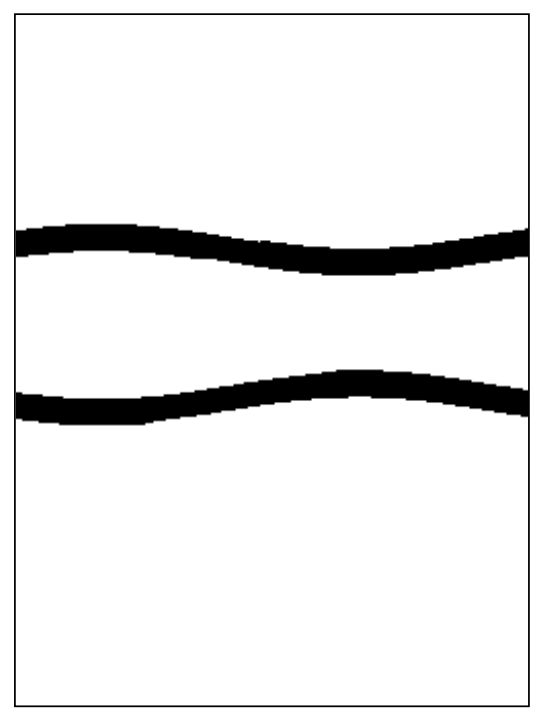

Figura 3 - Imagem binarizada obtida a partir da síntese do levantamento geofísico para o modelo 5.

\section{Resultados}

A técnica consiste em determinar automaticamente os parâmetros de amplitude, ângulo de fase, e coeficiente linear relacionados com uma senóide que representa a fratura em mapas de sondagem. Para o caso do modelo sintético 5 o algoritmo foi executado com 10 inicializações. A cada experimento o algoritmo zera os resultados e reinicializa a busca por todo o espaço de busca. Durante a execução as partículas alteram o seu estado (velocidade e posição). A cada iteração, a partícula é acelerada em direção à posição anterior da melhor partícula e a melhor posição global da mesma. A localização de cada partícula no enxame é afetada pela posição mais favorável durante o seu movimento, o que se convencionou chamar de experiência individual ou de avaliação da localização, que analisa a posição mais vantajosa do grupo (Figura 4).

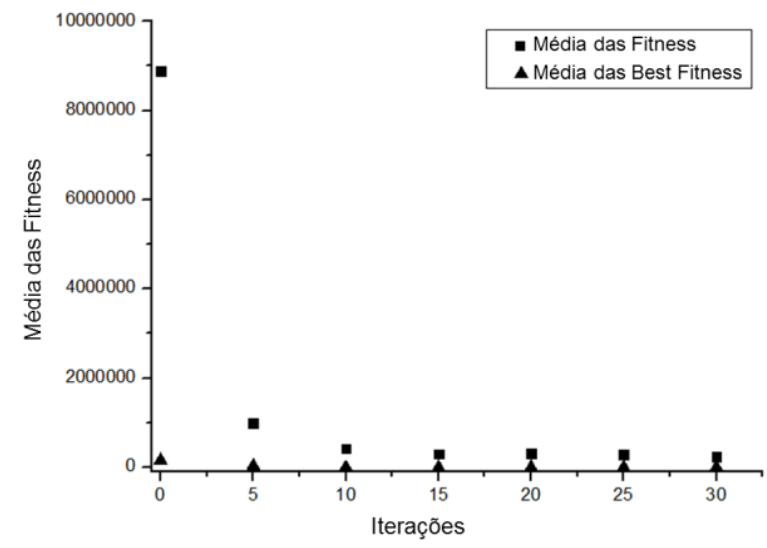

Figura 4 - Convergência do algoritmo PSO associado com o Método Multistart para a detecção automática das duas fraturas do modelo sintético 5 (Figura 2).
$\mathrm{Na}$ Tabela 4, pode-se observar os melhores parâmetros encontrados para as duas fraturas (Figura 2), de acordo com a aplicação do algoritmo PSO associado com o Método Multistart. O coeficiente linear, da inicialização 1, corresponde a primeira fratura (Figura 5). Já a segunda fratura foi detectada pelo coeficiente angular da inicialização 2 (Figura 6).

\begin{tabular}{c|l|c}
\hline Paramêntros & Inicialização 1 & Inicialização 2 \\
\hline Amplitude & 1.000000 & 1.000000 \\
\hline Ângulo de Fase & 4.040338 & 6.283185 \\
\hline Ceficiente Linear & 113.312270 & 186.250823 \\
\hline Best Fitness Final & 0.000000 & 0.000000 \\
\hline Fitness Média Final & 200033.800000 & 300003.900000 \\
\hline
\end{tabular}

Tabela 4 - Resultados obtidos com a aplicação do PSO associado com o Método Multistart para a detecção das duas fraturas do modelo sintético 5 .

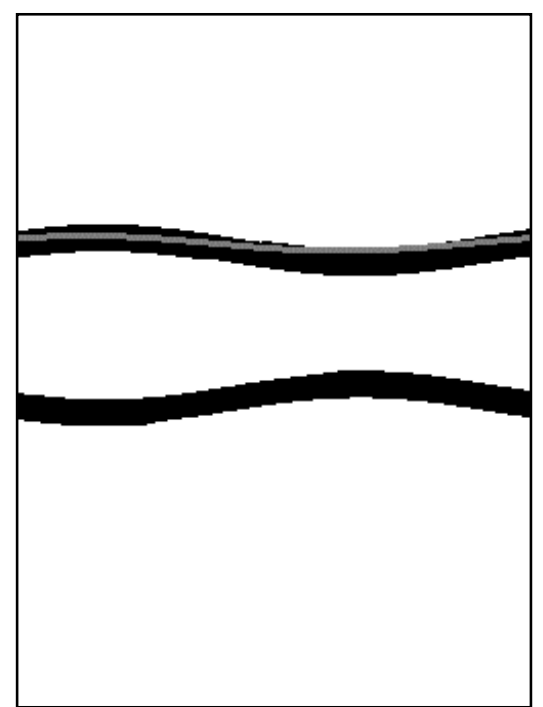

Figura 5 - Sobreposição entre a imagem da Figura 3 com a senóide gerada com os melhores parâmetros encontrados para a primeira fratura para o modelo sintético 5 (em tons de cinza). 


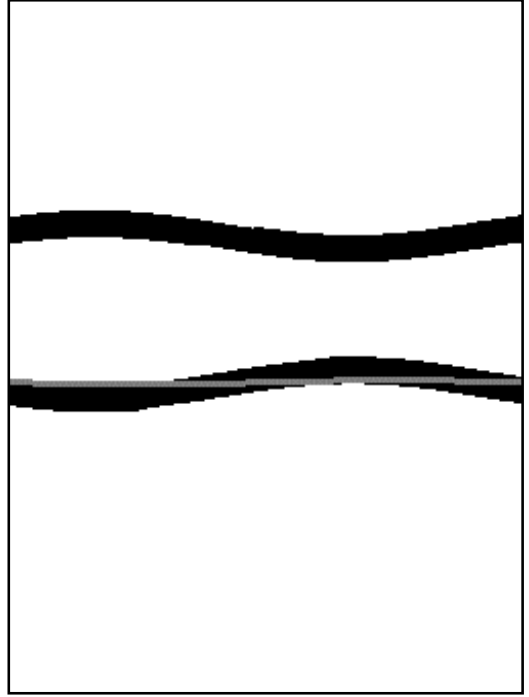

Figura 6 - Sobreposição entre a imagem da Figura 3 com a senóide gerada com os melhores parâmetros encontrados para a segunda fratura para o modelo sintético 5 (em tons de cinza).

As senóides geradas com os parâmetros encontrados com o método de combinar as fraturas representadas nas imagens do modelo sintético comprovaram a viabilidade do método (Figuras 5 e 6).

\section{Discussão e Conclusões}

O objetivo deste estudo é a implementação e testes de métodos de inteligência computacional (meta-heurísticas de otimização) para processamento e análise de perfis geofísicos, de forma a executar reconhecimento de padrões, desenvolvimento de capacidade de generalização do problema da perfilagem de poços, bem como a automatização desse processo.

Em Palheta et al. (2013) foram realizados teste, com o algoritmo PSO, para localizar os pontos de mínimo com uma única função objetivo, de modo que, o método combinava as fraturas representadas nas imagens do poço (modelo real) com cada solução candidata.

No presente trabalho, assim como em Palheta et al. (2013), cada uma das soluções candidatas é uma partícula com três coordenadas (amplitude, ângulo de fase e o coeficiente linear; parâmetros que descrevem uma senóide em um plano). Desta forma, o método de combinar as fraturas representadas na imagem do modelo sintético (Figura 2) com cada solução candidata é avaliada de acordo com as funções de fitnesses que avalia 0 ajuste da senóide em relação às fraturas que serão exibidas nas imagens (Figura 5 e 6), comprovando a viabilidade do método, pois, os parâmetros da senóde que melhor coincidem com as fraturas foram determinados. Assim, as sobreposições entre as curvas encontradas automaticamente, usando o algoritmo PSO associado com o Método Multistart, e a imagem original (Figura 2) demonstram o sucesso na utilização da técnica.

\section{Agradecimentos}

Ao CNPq (Conselho Nacional de Desenvolvimento Científico e Tecnológico, Ministério de Ciência e Tecnologia, Brasil).

\section{Referências}

Cardoso, T.N.; Meneses, A. A. M. . Otimização com Enxame de Partículas - Validação e Aplicação à FunçãoMultimodal de Himmelblau. In: XI ENCONTRO DE MODELAGEM COMPUTACIONAL, 2008, Volta Redonda. XI Encontro de Modelagem Computacional, 2008.

Eberhart, R.C., and Kennedy, J., 1995, A new optimizer using particles swarm theory: Proceedings of the IEEE sixth international symposium on micro machine and human science, Nagoya, Japan. 131p

Ellis, D.V. \& Singer, J.M. Earth logging for earth scientist. $2^{a}$ ed., New York, Springer Verlag, 2007.

Kennedy, J., and Eberhart, R.C., 2001, Swarm Intelligence: Morgan Kaufmann Publishers, USA.

Meneses, A. A. M, Machado, M. D., and Schirru, R., 2009. Particle Swarm Optimization applied to the nuclear reload problem of a pressurized water reactor. Progress in Nuclear Energy 51, 319-326.

Palheta, D. B; Guerra; C. E; Sacco, W. F; Meneses, A. A. M. .Automated Fracture Detection With Well Logs Using Global Optimization. In: 13th International Congress of the Brazilian Geophysical Society \& EXPOGEf, 2013, Rio de Janeiro.

Ugray, Zsolt, Leon Lasdon, John C. Plummer, Fred Glover, James Kelly, and Rafael Martí. Scatter Search and Local NLP Solvers: A Multistart Framework for Global Optimization. INFORMS Journal on Computing, Vol. 19, No. 3, 2007, pp. 328-340. 